Electronic Supporting Information for:

\title{
Pressure-Enhanced Environment Effects in Ferrocene Phases
}

Damian Paliwoda, ${ }^{*+, \neq, \S}$ Michael Hanfland, ${ }^{\ddagger}$ and Andrzej Katrusiak ${ }^{*+}$

${ }^{+}$Faculty of Chemistry, Adam Mickiewicz University, Uniwersytetu Poznańskiego 8, 61-614

Poznań, Poland

${ }^{\ddagger}$ European Synchrotron Radiation Facility, B.P. 220, 38043 Grenoble, France

${ }^{\S}$ SOLARIS National Synchrotron Radiation Centre, Jagiellonian University, Czerwone Maki

98, 30-392 Kraków, Poland

*Corresponding Authors:

Andrzej Katrusiak, e-mail: katran@amu.edu.pl;

Damian Paliwoda, e-mail: damian.paliwoda@gmail.com,

Contents

1. Experimental details.

2. Figure S1. Molecular volume of ferrocene phases I and I' compressed at 296, 180, 120 and $90 \mathrm{~K}$.

3. Figure S2. Unit-cell dimensions of ferrocene phases I and I' compressed at 296, 180, 120 and $90 \mathrm{~K}$.

4. Figure S3. The unit-cell parameters $b / c$ ratio plotted as a function of pressure - as shown in the plot in the main manuscript in Figure 2. The horizontal dashed line marks the asymptotic $b=c$, not reached within this experiment to $40 \mathrm{GPa}$.

5. Table S1. Selected synchrotron crystal data and structure refinements details for compression measurements of ferrocene crystals, phases I and I'.

6. Table S2. Unit-cell parameters of ferrocene crystals at $180 \mathrm{~K}$ and elevated pressures.

7. Table S3. Unit-cell parameters of ferrocene crystals at $120 \mathrm{~K}$ and elevated pressures.

8. Table S4. Unit-cell parameters of ferrocene crystals at $90 \mathrm{~K}$ and elevated pressures. 


\section{Experimental details:}

A series of room- and low-temperature high-pressure diffraction experiments have been performed at ID09A synchrotron beamline (ESRF) using the parallel monochromatic X-ray beam $(\mathrm{E}=30 \mathrm{keV}, \lambda=$ $0.4130 \AA$ ) focused to $30 \times 30 \mu \mathrm{m}^{2}$ on the sample. We used a membrane driven high-pressure cell equipped with Boehler-Almax seats and diamond-anvil design, allowing the cone windows opening of $64^{\circ}$. The culet diameter was $600 \mu \mathrm{m}$. The sample was loaded together with He as the pressure transmitting medium into a hole in a stainless-steel gasket. Room-temperature single-crystal data were collected through the vertical $\omega$-axis rotations with an integrated step scan of $0.5^{\circ}$ and a counting time of $1 \mathrm{~s}$ per frame up to $38.7 \mathrm{GPa}$. Low-temperature data at 180,120 and $90 \mathrm{~K}$ were collected using membrane-driven diamond anvil cell mounted into the cryostat, in the pressure range from $1 \mathrm{GPa}$ up to $8 \mathrm{GPa}$. Diffraction intensities were recorded with a Mar555 flat-panel detector. The diffraction data were processed and analysed with CrysAlisPro-171.38.41 software. Experimental data were not corrected for absorption because of its low value owing to the high energy of the X-ray beam. Frames have been rescaled using Friedel diffraction pairs to correct for variable diffracting volume as a function of the $\omega$ position. The pressure was measured with the ruby-fluorescence method according to the nonlinear hydrostatic calibration. ${ }^{1}$ The low-temperature data were insufficient for detailed structural refinements, however, the pressure evolution of the unit-cell parameters were successfully extracted. Structural analyses for room-temperature high-pressure data were performed using program Shelx. ${ }^{2}$ The crystallographic information of ferrocene crystal structures has been deposited in CIF format at the Cambridge Crystallographic Database Centre and is provided in the ESI, $\dagger$ CCDC 1936387-1936404.

(1) Mao, H.-K.; Xu, J.; Bell, P. M.; J. Geophys. Res., B, 1986, 91, 46973-46976.

(2) Sheldrick, G. M.; Acta Crystallogr. Sect. C, 2015, 71, 3-8. 

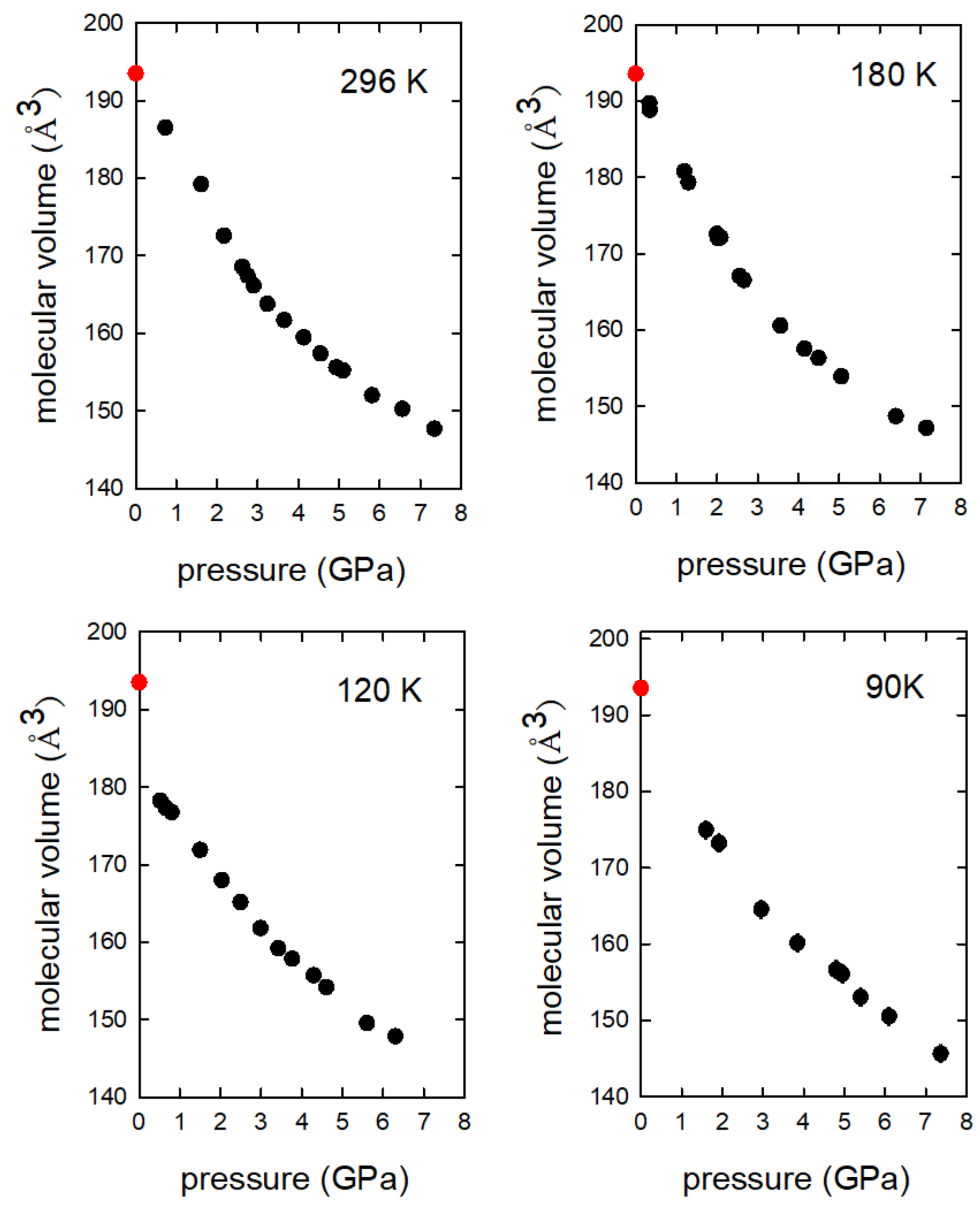

Figure S1. Molecular volume of ferrocene phases I and I' compressed at 296, 180, 120 and $90 \mathrm{~K}$. 

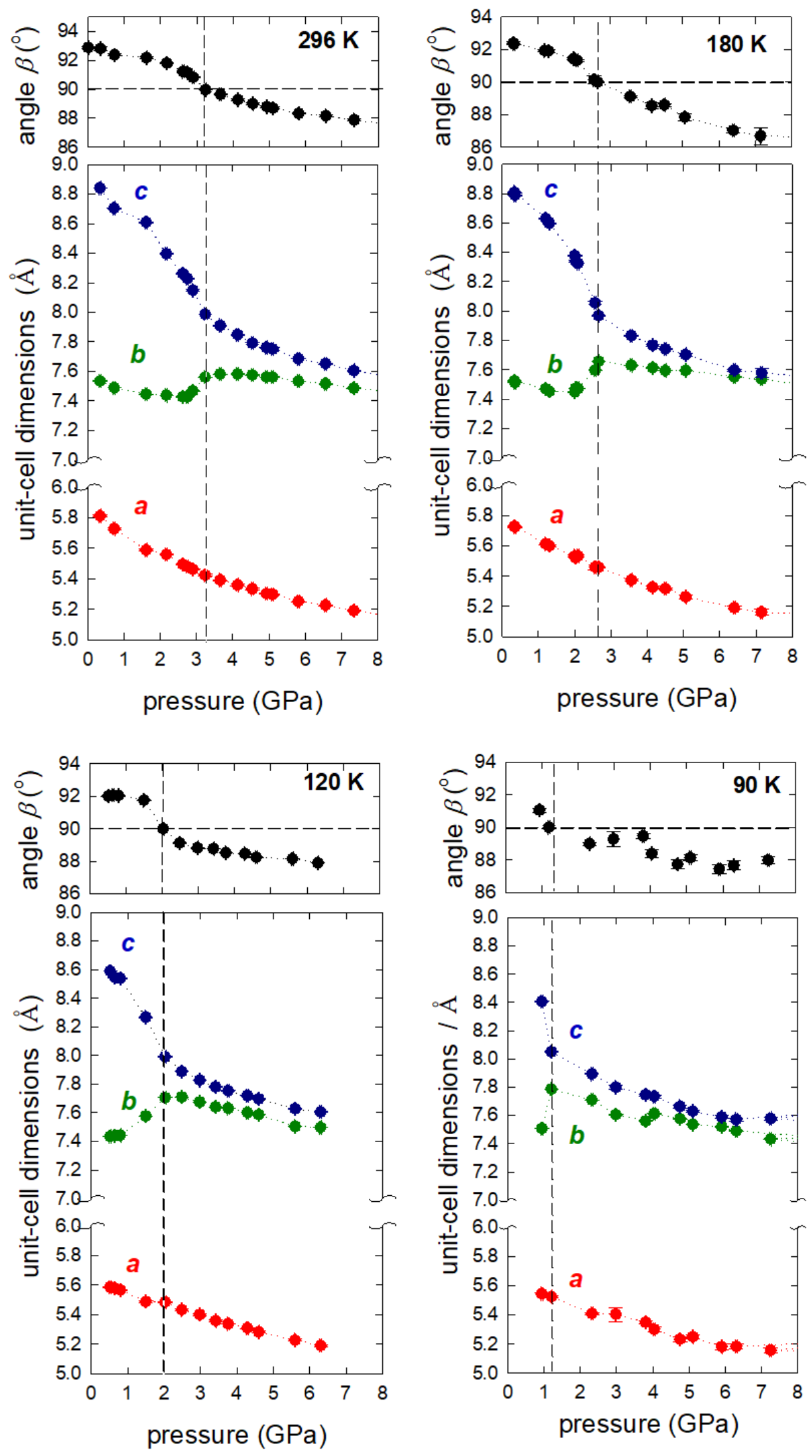

Figure S2. Unit-cell dimensions of ferrocene phases I and I' compressed at 296, 180, 120 and $90 \mathrm{~K}$. 


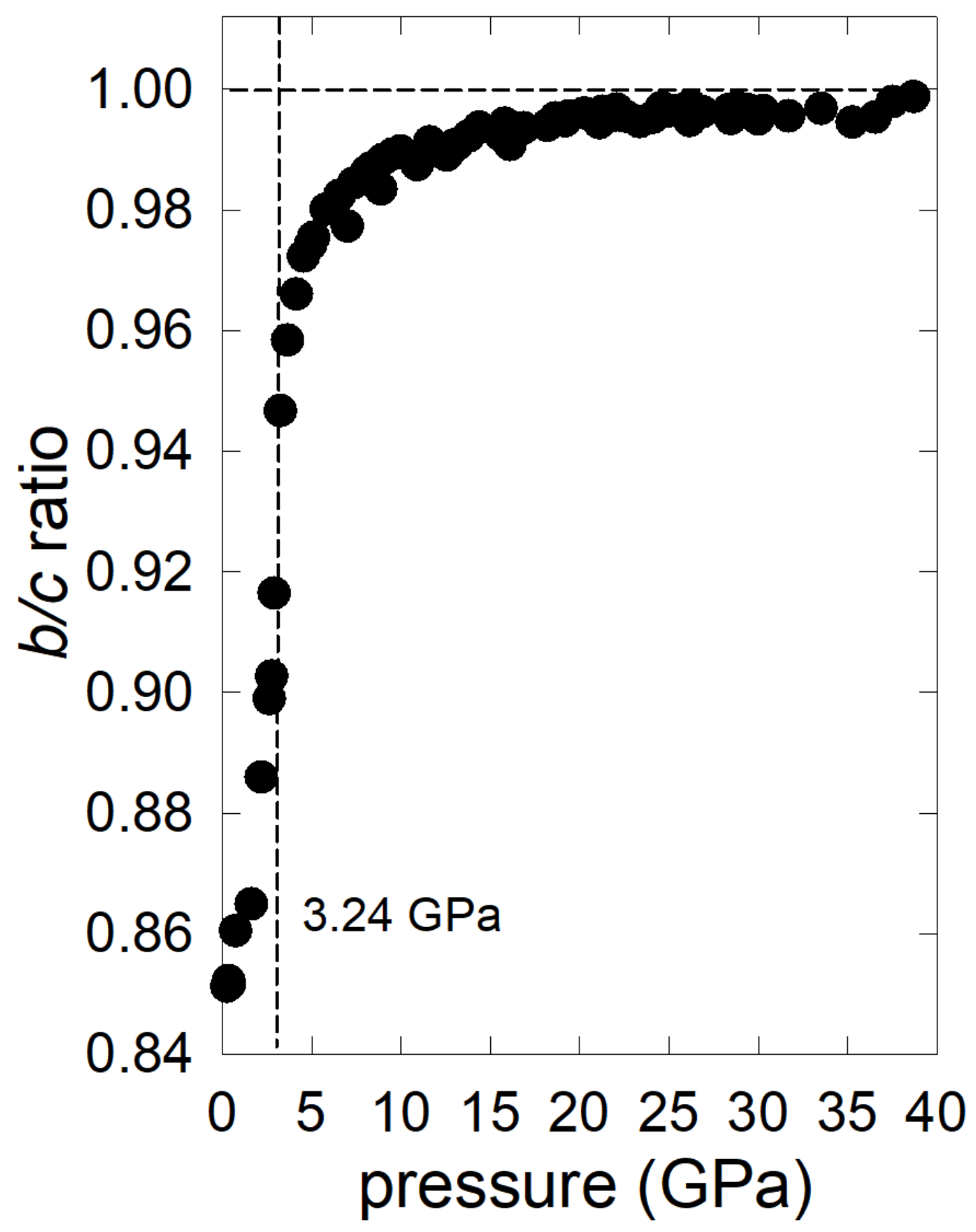

Figure S3. The unit-cell parameters $b / c$ ratio plotted as a function of pressure - as shown in the plot in the main manuscript in Figure 2. The horizontal dashed line marks the asymptotic $b=c$, not reached within this experiment to $40 \mathrm{GPa}$. 
Table S1. Selected synchrotron crystal data and structure refinements details for compression measurements of ferrocene crystals, phases I and I'.

\begin{tabular}{|c|c|c|c|c|c|c|c|c|c|c|}
\hline polymorph & $\mathrm{I}$ & l' & I' & $\mathrm{l}^{\prime}$ & I' & I' & I' & $\mathrm{l}^{\prime}$ & I' & l' \\
\hline $\begin{array}{l}\text { temperature, } \mathrm{K} \\
\text { pressure } \\
\text { formula }\end{array}$ & $\begin{array}{c}296(2) \\
0.24 \mathrm{GPa} \\
\mathrm{FeC}_{10} \mathrm{H}_{10}\end{array}$ & $\begin{array}{c}296(2) \\
7.00 \mathrm{GPa} \\
\mathrm{FeC}_{10} \mathrm{H}_{10}\end{array}$ & $\begin{array}{c}296(2) \\
8,87 \mathrm{GPa} \\
\mathrm{FeC}_{10} \mathrm{H}_{10}\end{array}$ & $\begin{array}{c}296(2) \\
10.90 \mathrm{GPa} \\
\mathrm{FeC}_{10} \mathrm{H}_{10}\end{array}$ & $\begin{array}{c}296(2) \\
13.12 \mathrm{GPa} \\
\mathrm{FeC}_{10} \mathrm{H}_{10}\end{array}$ & $\begin{array}{c}296(2) \\
16.10 \mathrm{GPa} \\
\mathrm{FeC}_{10} \mathrm{H}_{10}\end{array}$ & $\begin{array}{c}296(2) \\
19.18 \mathrm{GPa} \\
\mathrm{FeC}_{10} \mathrm{H}_{10}\end{array}$ & $\begin{array}{c}296(2) \\
21.10 \mathrm{GPa} \\
\mathrm{FeC}_{10} \mathrm{H}_{10}\end{array}$ & $\begin{array}{c}296(2) \\
23.35 \mathrm{GPa} \\
\mathrm{FeC}_{10} \mathrm{H}_{10}\end{array}$ & $\begin{array}{c}296(2) \\
26.15 \mathrm{GPa} \\
\mathrm{FeC}_{10} \mathrm{H}_{10}\end{array}$ \\
\hline $\mathrm{fw}, \mathrm{g} / \mathrm{mol}$ & 186.04 & 186.04 & 186.04 & 186.04 & 186.04 & 186.04 & 186.04 & 186.04 & 186.04 & 186.04 \\
\hline $\begin{array}{l}\text { crystal size, } \mathrm{mm} \\
\text { crystal colour }\end{array}$ & $\begin{array}{c}0.05 \times 0.04 \times 0.02 \\
\text { orange }\end{array}$ & $\begin{array}{c}0.05 \times 0.04 \times 0.02 \\
\text { orange }\end{array}$ & $\begin{array}{c}0.05 \times 0.04 \times 0.02 \\
\text { orange }\end{array}$ & $\begin{array}{c}0.5 \times 0.04 \times 0.02 \\
\text { orange }\end{array}$ & $\begin{array}{c}0.05 \times 0.04 \times 0.02 \\
\text { orange }\end{array}$ & $\begin{array}{c}0.05 \times 0.04 \times 0.02 \\
\text { orange }\end{array}$ & $\begin{array}{c}0.05 \times 0.04 \times 0.02 \\
\text { orange }\end{array}$ & $\begin{array}{c}0.05 \times 0.04 \times 0.02 \\
\text { orange }\end{array}$ & $\begin{array}{c}0.05 \times 0.04 \times 0.02 \\
\text { orange }\end{array}$ & $\begin{array}{c}0.05 \times 0.04 \times 0.02 \\
\text { orange }\end{array}$ \\
\hline crystal system & monoclinic & monoclinic & monoclinic & monoclinic & monoclinic & monoclinic & monoclinic & monoclinic & monoclinic & monoclinic \\
\hline space group, $Z$ & $P 2_{1} / n, 2$ & $P 2_{1} / n, 2$ & $P 2_{1} / n, 2$ & $P 2_{1} / n, 2$ & $P 2_{1} / n, 2$ & $P 2_{1} / n, 2$ & $P 2, / n, 2$ & $P 2_{1} / n, 2$ & $P 21 / n, 2$ & $P 2 / n, 2$ \\
\hline a, $\AA$, & $5.829(3)$ & $5.219(3)$ & $5.147(2)$ & $5.088(2)$ & $5.0135(13)$ & $4.9494(15)$ & $4.886(2)$ & $4.8528(16)$ & $4.8188(14)$ & $4.7770(17)$ \\
\hline$b, \AA$ & $7.5446(5)$ & $7.4846(6)$ & $7.4303(4)$ & $7.3808(4)$ & $7.3302(3)$ & $7.2600(3)$ & $7.2013(4)$ & $7.1660(3)$ & $7.1267(3)$ & $7.0864(4)$ \\
\hline$c, \AA$ & $8.8627(15)$ & $7.6584(16)$ & $7.5553(12)$ & $7.4747(11)$ & $7.3984(11)$ & $7.3172(11)$ & $7.2394(14)$ & $7.2062(13)$ & $7.1653(12)$ & $7.1235(12)$ \\
\hline$\beta,{ }^{\circ}$ & $92.78(4)$ & $88.32(4)$ & $87.78(3)$ & $87.36(3)$ & $86.81(2)$ & $86.47(3)$ & $85.97(3)$ & $85.78(3)$ & $85.65(3)$ & $85.67(3)$ \\
\hline $\mathrm{V}, \AA^{3}$ & 389.3(2) & $299.00(16)$ & $288.71(12)$ & $280.40(12)$ & $271.47(8)$ & $262.43(9)$ & $254.12(12)$ & 249.92(9) & $245.36(8)$ & $240.45(10)$ \\
\hline$\rho, \mathrm{g} / \mathrm{cm}^{3}$ & 1.587 & 2.066 & 2.140 & 2.203 & 2.276 & 2.354 & 2.431 & 2.472 & 2.518 & 2.569 \\
\hline$\mu, \mathrm{mm}^{-1}$ & 1.855 & 2.416 & 2.502 & 2.576 & 2.661 & 2.752 & 2.842 & 2.890 & 2.944 & 3.004 \\
\hline$F(000)$ & 192 & 192 & 192 & 192 & 192 & 192 & 192 & 192 & 192 & 192 \\
\hline$\theta$ range $^{\circ}$ & $3.55-37.56$ & 5.33- 37.99 & $3.85-37.76$ & 5.4637 .43 & $5.54-38.54$ & $5.60-36.83$ & $5.64-36.71$ & $5.67-39.41$ & $5.70-37.14$ & $5.74-37,16$ \\
\hline \multirow{3}{*}{ limiting indices } & $-5 \Rightarrow h \Rightarrow 5$ & $-5 \Rightarrow h \Rightarrow 5$ & $-5 \Rightarrow h \Rightarrow 5$ & $-4 \Rightarrow h \Rightarrow 5$ & $-5 h \Rightarrow 5$ & $-5 \Rightarrow h \Rightarrow 5$ & $-4 \Rightarrow h \Rightarrow 5$ & $-4 \Rightarrow h \Rightarrow 5$ & $-5 \Rightarrow h \Rightarrow 4$ & $-4 \Rightarrow h \Rightarrow 4$ \\
\hline & $-10 \Rightarrow k \Rightarrow 10$ & $-10 \Rightarrow k \Rightarrow 10$ & $-10 \Rightarrow k \Rightarrow 10$ & $-10 \Rightarrow k \Rightarrow 10$ & $-10 \Rightarrow k \Rightarrow 10$ & $-10 \Rightarrow k \Rightarrow 10$ & $-10 \Rightarrow k \Rightarrow 10$ & $-9 \Rightarrow k \Rightarrow 10$ & $-9 \Rightarrow k \Rightarrow 10$ & $-9 \Rightarrow k \Rightarrow 9$ \\
\hline & $-11 \Rightarrow \mid \Rightarrow 10$ & $-9 \Rightarrow \mid \Rightarrow 8$ & $-8 \Rightarrow \mid \Rightarrow 9$ & $-8 \Rightarrow \mid \Rightarrow 9$ & $-9 \Rightarrow \mid \Rightarrow 8$ & $-8 \Rightarrow \mid \Rightarrow 8$ & $-8 \Rightarrow \mid \Rightarrow 8$ & $-8 \Rightarrow \mid \Rightarrow 8$ & $-8 \Rightarrow \mid \Rightarrow 8$ & $-8 \Rightarrow \mid \Rightarrow 8$ \\
\hline reflns collected & 1070 & 816 & 846 & 842 & 765 & 723 & 720 & 717 & 710 & 688 \\
\hline Rint & 0.032 & 0.030 & 0.020 & 0.022 & 0.023 & 0.022 & 0.017 & 0.018 & 0.016 & 0.017 \\
\hline data/parameters & $467 / 53$ & $345 / 52$ & $340 / 53$ & $340 / 52$ & $310 / 52$ & $595 / 52$ & $284 / 47$ & $281 / 52$ & $272 / 52$ & $266 / 52$ \\
\hline GOF on $\mathrm{F}^{2}$ & 1.229 & 1.173 & 1.244 & 1.196 & 1.192 & 1.153 & 1.185 & 1.233 & 1.192 & 1.212 \\
\hline$R_{1}[\mathrm{I}>2 \sigma(\mathrm{I})]$ & 0.0671 & 0.0643 & 0.0533 & 0.0404 & 0.0667 & 0.0550 & 0.0585 & 0.0544 & 0.0482 & 0.0395 \\
\hline$R_{1}$ (all data) & 0.0818 & 0.0775 & 0.0603 & 0.0442 & 0.0700 & 0.0594 & 0.0614 & 0.0561 & 0.0495 & 0.0403 \\
\hline $\begin{array}{l}w R_{2} \text { (all data) } \\
\text { completness to }\end{array}$ & 0.1871 & 0.1819 & 0.1486 & 0.1175 & 0.1955 & 0.1624 & 0.1843 & 0.1678 & 0.1470 & 0.01187 \\
\hline $\begin{array}{l}\text { theta max } \\
\text { Irgst diff peak, }\end{array}$ & $22.8 \%$ & $21.2 \%$ & $22.1 \%$ & $23.1 \%$ & $20.3 \%$ & $21.1 \%$ & $22.5 \%$ & $18.8 \%$ & $21.7 \%$ & $21.6 \%$ \\
\hline $\begin{array}{l}\mathrm{e} / \AA^{3} \\
\text { Irgst diff hole, }\end{array}$ & 0.555 & 0.591 & 0.435 & 0.654 & 0.886 & 0.666 & 0.591 & 0.664 & 0.578 & 0.465 \\
\hline $\mathrm{e} / \AA^{3}$ & -0.397 & -0.541 & -0.742 & -0.609 & -0.911 & -0.729 & -0.704 & -0.738 & -0.639 & -0.482 \\
\hline
\end{tabular}


Table S1. Selected synchrotron crystal data and structure refinements details for compression measurements of ferrocene crystals, phases I and I'.

\begin{tabular}{|c|c|c|c|c|c|c|c|c|}
\hline polymorph & I' & I' & $I^{\prime}$ & I' & $I^{\prime}$ & $I^{\prime}$ & I' & I' \\
\hline $\begin{array}{l}\text { temperature, } \mathrm{K} \\
\text { pressure } \\
\text { formula }\end{array}$ & $\begin{array}{c}296(2) \\
28.46 \mathrm{GPa} \\
\mathrm{FeC}_{10} \mathrm{H}_{10}\end{array}$ & $\begin{array}{c}296(2) \\
30,00 \mathrm{GPa} \\
\mathrm{FeC}_{10} \mathrm{H}_{10}\end{array}$ & $\begin{array}{c}296(2) \\
31,695 \mathrm{GPa} \\
\mathrm{FeC}_{10} \mathrm{H}_{10}\end{array}$ & $\begin{array}{c}296(2) \\
33.52 \mathrm{GPa} \\
\mathrm{FeC}_{10} \mathrm{H}_{10}\end{array}$ & $\begin{array}{c}296(2) \\
35.26 \mathrm{GPa} \\
\mathrm{FeC}_{10} \mathrm{H}_{10}\end{array}$ & $\begin{array}{c}296(2) \\
36.545 \mathrm{GPa} \\
\mathrm{FeC}_{10} \mathrm{H}_{10}\end{array}$ & $\begin{array}{c}296(2) \\
37,51 \mathrm{GPa} \\
\mathrm{FeC}_{10} \mathrm{H}_{10}\end{array}$ & $\begin{array}{c}296(2) \\
38,70 \mathrm{GPa} \\
\mathrm{FeC}_{10} \mathrm{H}_{10}\end{array}$ \\
\hline $\begin{array}{l}\mathrm{fw}, \mathrm{g} / \mathrm{mol} \\
\text { crystal size, } \mathrm{mm} \\
\text { crystal colour } \\
\text { crystal system } \\
\text { space group, } Z\end{array}$ & $\begin{array}{c}186.04 \\
0.05 \times 0.04 \times 0.02 \\
\text { orange } \\
\text { monoclinic } \\
P 2_{1} / n, 2\end{array}$ & $\begin{array}{c}186.04 \\
0.05 \times 0.04 \times 0.02 \\
\text { orange } \\
\text { monoclinic } \\
P 2_{1} / n, 2\end{array}$ & $\begin{array}{c}186.04 \\
0.05 \times 0.04 \times 0.02 \\
\text { orange } \\
\text { monoclinic } \\
P 2_{1} / n, 2\end{array}$ & $\begin{array}{c}186.04 \\
0.5 \times 0.04 \times 0.02 \\
\text { orange } \\
\text { monoclinic } \\
P 2_{1} / n, 2\end{array}$ & $\begin{array}{c}186.04 \\
0.05 \times 0.04 \times 0.02 \\
\text { orange } \\
\text { monoclinic } \\
P 2_{1} / n, 2\end{array}$ & $\begin{array}{c}186.04 \\
0.05 \times 0.04 \times 0.02 \\
\text { orange } \\
\text { monoclinic } \\
P 2_{1} / n, 2\end{array}$ & $\begin{array}{c}186.04 \\
0.05 \times 0.04 \times 0.02 \\
\text { orange } \\
\text { monoclinic } \\
P 2_{1} / n, 2\end{array}$ & $\begin{array}{c}186.04 \\
0.05 \times 0.04 \times 0.02 \\
\text { orange } \\
\text { monoclinic } \\
P 2_{1} / n, 2\end{array}$ \\
\hline$a, \AA$ & $4.7443(15)$ & $4.7296(16)$ & $4.7048(14)$ & $4.6874(19)$ & $4.664(3)$ & $4.650(3)$ & $6.9553(4)$ & $4.6322(16)$ \\
\hline$b, \AA$ & $7.0532(3)$ & $7.0364(3)$ & $7.0179(3)$ & $7.0015(4)$ & $6.9728(6)$ & $6.9615(6)$ & $6.9553(4)$ & $6.9487(4)$ \\
\hline$c, \AA$ & $7.0879(12)$ & $7.0713(13)$ & $7.0491(11)$ & $7.0239(16)$ & 7.011(2) & $6.994(2)$ & $6.9696(15)$ & $6.9573(15)$ \\
\hline$\beta,{ }^{\circ}$ & $85.27(3)$ & $85.03(3)$ & $84.77(3)$ & $84.54(4)$ & $84.60(5)$ & $84.42(5)$ & $84.19(3)$ & $84.01(3)$ \\
\hline $\mathrm{V}, \AA^{3}$ & $236.37(9)$ & $234.44(9)$ & $231.78(8)$ & $229.47(11)$ & $227.01(16)$ & $225.32(14)$ & 223.92(9) & $222.72(9)$ \\
\hline$\rho, \mathrm{g} / \mathrm{cm}^{3}$ & 2.614 & 2.635 & 2.666 & 2.203 & 2.722 & 2.742 & 2.759 & 2.774 \\
\hline$\mu, \mathrm{mm}^{-1}$ & 3.056 & 3.081 & 3.116 & 2.576 & 3.182 & 3.205 & 3.225 & 3.243 \\
\hline$F(000)$ & 192 & 192 & 192 & 192 & 192 & 192 & 192 & 192 \\
\hline$\theta$ range, ${ }^{\circ}$ & $5.77-36.83$ & $5.77-37.38$ & 5.79- 37.49 & $5.80-37.87$ & $5.83-36.53$ & $5.84-36.59$ & $5.84-38.15$ & $5.85-40.00$ \\
\hline limiting indices & $\begin{array}{l}-4 \Rightarrow h \Rightarrow 5 \\
-9 \Rightarrow k \Rightarrow 9 \\
-8 \Rightarrow l \Rightarrow 8\end{array}$ & $\begin{array}{c}-5 \Rightarrow h \Rightarrow 4 \\
-9 \Rightarrow k \Rightarrow 9 \\
-8 \Rightarrow \mid \Rightarrow 8\end{array}$ & $\begin{aligned}-5 \Rightarrow h \Rightarrow 4 \\
-9 \Rightarrow k \Rightarrow 9 \\
-8 \Rightarrow l \Rightarrow 9\end{aligned}$ & $\begin{array}{l}-4 \Rightarrow h \Rightarrow 5 \\
-9 \Rightarrow k \Rightarrow 9 \\
-8 \Rightarrow \mid \Rightarrow 8\end{array}$ & $\begin{array}{c}-5 h \Rightarrow 5 \\
-10 \Rightarrow k \Rightarrow 10 \\
-9 \Rightarrow l \Rightarrow 8\end{array}$ & $\begin{array}{l}-4 \Rightarrow h \Rightarrow 4 \\
-9 \Rightarrow k \Rightarrow 9 \\
-7 \Rightarrow l \Rightarrow 8\end{array}$ & $\begin{array}{l}-5 \Rightarrow h \Rightarrow 4 \\
-9 \Rightarrow k \Rightarrow 9 \\
-8 \Rightarrow \mid \Rightarrow 8\end{array}$ & $\begin{array}{l}-5 \Rightarrow h \Rightarrow 4 \\
-9 \Rightarrow k \Rightarrow 9 \\
-8 \Rightarrow l \Rightarrow 8\end{array}$ \\
\hline reflns collected & 600 & 636 & 628 & 638 & 765 & 623 & 610 & 594 \\
\hline 1 & 0.0195 & 0.030 & 0.0146 & 0.0146 & 0.023 & 0.0312 & 0.0201 & 0.0255 \\
\hline data/parameters & $249 / 52$ & $258 / 52$ & $258 / 52$ & $261 / 52$ & $310 / 52$ & $250 / 52$ & $243 / 52$ & $281 / 52$ \\
\hline GOF on $\mathrm{F}^{2}$ & 1.207 & 1.127 & 1.244 & 1.164 & 1.322 & 1.185 & 1.204 & 1.167 \\
\hline$R_{1}[\mathrm{I}>2 \sigma(\mathrm{I})]$ & 0.0415 & 0.0478 & 0.0459 & 0.0540 & 0.0850 & 0.0653 & 0.0463 & 0.0575 \\
\hline$R_{1}$ (all data) & 0.0427 & 0.0493 & 0.0485 & 0.0566 & 0.0890 & 0.0727 & 0.0492 & 0.0632 \\
\hline $\begin{array}{l}w R_{2} \text { (all data) } \\
\text { completness to }\end{array}$ & 0.1271 & 0.1416 & 0.1445 & 0.1537 & 0.2463 & 0.1913 & 0.1294 & 0.1578 \\
\hline $\begin{array}{l}\text { theta max } \\
\text { Irgst diff peak, }\end{array}$ & $21.1 \%$ & $21.1 \%$ & $21.3 \%$ & $21.2 \%$ & $22.2 \%$ & $22.4 \%$ & $19.8 \%$ & $17.5 \%$ \\
\hline $\begin{array}{l}\mathrm{e} / \AA^{3} \\
\text { Irgst diff hole, }\end{array}$ & 0.391 & 0.471 & 0.435 & 0.532 & 1.169 & 0.707 & 0.478 & 0.585 \\
\hline $\mathrm{e} / \AA^{3}$ & -0.507 & -0.541 & -0.518 & -0.733 & -1.054 & -1.167 & -0.389 & -0.694 \\
\hline
\end{tabular}


Table S2. Unit cell-parameters of ferrocene crystals at $180 \mathrm{~K}$ and elevated pressures.

\begin{tabular}{l|c|c|c|c|c}
\hline $\begin{array}{l}\text { Pressure } \\
(\mathrm{GPa})\end{array}$ & $a(\AA)$ & $b(\AA)$ & $c(\AA)$ & $\beta\left(^{0}\right)$ & $V\left(\AA^{3}\right)$ \\
\hline 0.34 & $5.729(4)$ & $7.5245(7)$ & $8.809(4)$ & $92.41(7)$ & 377.6206 \\
0.35 & $5.723(5)$ & $7.5130(4)$ & $8.789(2)$ & $92.34(5)$ & 379.4038 \\
1.2 & $5.612(4)$ & $7.4691(6)$ & $8.629(3)$ & $91.93(6)$ & 361.5436 \\
1.3 & $5.601(5)$ & $7.4535(7)$ & $8.596(4)$ & $91.90(7)$ & 358.6584 \\
2 & $5.532(5)$ & $7.4492(7)$ & $8.378(4)$ & $91.44(7)$ & 345.1664 \\
2.02 & $5.522(4)$ & $7.474(2)$ & $8.339(5)$ & $91.39(5)$ & 344.0642 \\
2.09 & $5.534(4)$ & $7.4730(5)$ & $8.326(3)$ & $91.34(6)$ & 344.2152 \\
2.56 & $5.459(17)$ & $7.597(3)$ & $8.055(12)$ & $90.1(3)$ & 334.093 \\
2.66 & $5.458(2)$ & $7.6569(8)$ & $7.967(1)$ & $90(0)$ & 333.0564 \\
3.56 & $5.374(5)$ & $7.6307(6)$ & $7.832(3)$ & $89.11(7)$ & 321.131 \\
4.15 & $5.327(11)$ & $7.614(1)$ & $7.769(8)$ & $88.53(16)$ & 315.015 \\
4.5 & $5.317(6)$ & $7.5951(9)$ & $7.743(5)$ & $88.59(9)$ & 312.5878 \\
5.06 & $5.264(15)$ & $7.596(2)$ & $7.704(10)$ & $87.8(2)$ & 307.8078 \\
6.4 & $5.191(10)$ & $7.552(1)$ & $7.597(8)$ & $87.02(16)$ & 297.4068 \\
7.15 & $5.16(2)$ & $7.536(1)$ & $7.58(3)$ & $86.7(5)$ & 294.3608 \\
8.8 & $5.151(7)$ & $7.484(4)$ & $7.548(7)$ & $86.59(9)$ & 290.5918 \\
\hline
\end{tabular}

Table S3. Unit cell-parameters of ferrocene crystals at $120 \mathrm{~K}$ and elevated pressures.

\begin{tabular}{l|c|c|c|c|c}
\hline $\begin{array}{l}\text { Pressure } \\
(\mathrm{GPa})\end{array}$ & $a(\AA)$ & $b(\AA)$ & $c(\AA)$ & $\beta\left(^{0}\right)$ & $V\left(\AA^{3}\right)$ \\
\hline 0.45 & $5.586(3)$ & $7.4351(4)$ & $8.590(2)$ & $92.00(4)$ & 356.5 \\
0.65 & $5.577(6)$ & $7.4391(8)$ & $8.544(5)$ & $92.06(9)$ & 354.68 \\
0.8 & $5.566(5)$ & $7.4430(5)$ & $8.538(4)$ & $92.03(8)$ & 353.5 \\
1.5 & $5.488(5)$ & $7.5770(7)$ & $8.268(4)$ & $91.75(7)$ & 343.77 \\
2.03 & $5.484(5)$ & $7.7058(7)$ & $7.991(4)$ & $90(0)$ & 335.98 \\
2.49 & $5.432(4)$ & $7.7101(6)$ & $7.888(3)$ & $89.12(6)$ & 330.3 \\
2.98 & $5.400(6)$ & $7.6753(7)$ & $7.827(3)$ & $88.82(7)$ & 323.6 \\
3.42 & $5.358(7)$ & $7.6402(9)$ & $7.780(3)$ & $88.78(9)$ & 318.4 \\
3.76 & $5.337(2)$ & $7.6314(7)$ & $7.754(1)$ & $88.52(5)$ & 315.7 \\
4.29 & $5.308(5)$ & $7.6012(5)$ & $7.720(2)$ & $88.47(6)$ & 311.4 \\
4.6 & $5.283(6)$ & $7.5872(8)$ & $7.697(3)$ & $88.24(8)$ & 308.4 \\
5.6 & $5.227(8)$ & $7.505(1)$ & $7.628(4)$ & $88.15(1)$ & 299.1 \\
6.3 & $5.190(7)$ & $7.4978(9)$ & $7.605(3)$ & $87.90(9)$ & 295.7 \\
\hline
\end{tabular}


Table S4. Unit cell-parameters of ferrocene crystals at $90 \mathrm{~K}$ and elevated pressures.

\begin{tabular}{l|c|c|c|c|c}
\hline $\begin{array}{l}\text { Pressure } \\
(\mathrm{GPa})\end{array}$ & $a(\AA)$ & $b(\AA)$ & $c(\AA)$ & $\beta\left(^{0}\right)$ & $V\left(\AA^{3}\right)$ \\
\hline 0.94 & $5.544(9)$ & $7.508(1)$ & $8.406(5)$ & $91.06(1)$ & 349.86 \\
1.2 & $5.525(8)$ & $7.787(2)$ & $8.051(4)$ & $90(0)$ & 346.4 \\
2.32 & $5.409(7)$ & $7.7094(8)$ & $7.893(4)$ & $88.99(9)$ & 329.1506 \\
2.98 & $5.40(5)$ & $7.606(3)$ & $7.79(1)$ & $89.3(5)$ & 320.553 \\
3.8 & $5.34(1)$ & $7.561(2)$ & $7.746(7)$ & $89.5(2)$ & 313.2442 \\
4.04 & $5.30(2)$ & $7.613(2)$ & $7.735(9)$ & $88.4(2)$ & 312.0684 \\
4.75 & $5.23(2)$ & $7.577(2)$ & $7.66(1)$ & $87.7(3)$ & 303.6662 \\
5.1 & $5.25(1)$ & $7.537(2)$ & $7.630(7)$ & $88.1(2)$ & 301.7562 \\
5.9 & $5.18(2)$ & $7.520(3)$ & $7.59(1)$ & $87.4(3)$ & 295.4858 \\
6.3 & $5.18(2)$ & $7.491(2)$ & $7.57(1)$ & $87.6(3)$ & 293.7604 \\
7.25 & $5.15(2)$ & $7.433(2)$ & $7.58(1)$ & $87.9(2)$ & 290.3928 \\
\hline
\end{tabular}

